\title{
Prevention of dental anomalies and dental caries in children with diabetes
}

\begin{abstract}
F. R. Kamalova ${ }^{1}$
${ }^{1}$ Bukhara state medical Institute

Abstract: Among diseases of the endocrine system in children, diabetes mellitus is the most significant, severe and requiring special attention pathology, diabetes can occur at any age, occurs in infants, preschool children and adolescents. Children's diabetes, as well as in adults, has a chronic, lifelong course and often causes severe complications, so it is very important to detect the disease in a timely manner and take the necessary measures for its adequate treatment.

Diabetes mellitus is a global problem of medical science and health care worldwide. Diabetes causes significant damage to the health of people of almost all Nations and all ages .

Worldwide, there is an increase in the prevalence of type 1 and type 2 diabetes among children. In this regard, dentists who provide dental care for children need knowledge and skills about the impact of diabetes on dental health, the relationship and mutual influence of dental pathology and diabetes in children.
\end{abstract}

Keywords: Diabetes mellitus, dental pathology, endocrine system, dental anomalies.

\section{Introduction}

Increase the effectiveness of primary prevention of major dental diseases in children with diabetes who are under medical supervision in the endocrinological dispensary of Bukhara.

The tasks were to determine the prevalence and intensity of dental caries, dental anomalies, the prevalence of diseases, periodontal tissues. Develop "Healthy tooth" lessons for children and adolescents with various degrees of endocrinological diseases and implement them in the educational process.

\section{Materials and methods}

We examined 60 children from 5 to 17 years of age in the regional endocrinological dispensary of Bukhara And the children's Department with diabetes mellitus .

All the children were divided into 3 groups:

I - group consisted of children with temporary occlusion (12)

II - group consisted of children with exchangeable bite (20)

III-group consisted of children with permanent dentition (28).

All the children we took into account the following performance tests: blood sugar level, age, health index, the RMA index and the indices KP,KPU KP, KPU. At the same time, a comprehensive study of the oral cavity was conducted, which included identifying patients ' complaints, collecting anamnesis, and visual examination.

The diagnosis of diabetes mellitus was established based on the study of anamnestic data, clinical examination, laboratory, x-ray and other research methods. Children with a mild form of the disease were not identified, most children (38 children) had a severe form of diabetes, they had a tendency to ketoacidosis, and a history of comatose States. According to parents, it was noted that $50 \%$ of children have never been examined by a pediatric dentist.

During the study, most children were found to have concomitant diseases, such as gastrointestinal diseases-chronic cholecystitis, chronic hepatitis, chronic pancreatitis, chronic anemia-II degree, chronic pyelonephritis and visual disorders.

To assess the condition of periodontal tissues, the following indices were used: the gingivitis index (PMA), the intensity of the carious process was determined using the CPI index, CPI+CP, CP.

To determine the hygienic state of the oral cavity, the method of J. C. Green and J. R. Vermillion (1964) was used.

A comprehensive study was conducted to assess anomalies of the dentition system. It included detection of the type of breathing, swallowing function, chewing,presence of crowding of teeth, diastema, the state of permanent 6 teeth, the state of the child's chewing muscles, the usual position during sleep, the presence of bad habits. In the external examination determined by the proportionality and symmetry of the contours of the face, when intraoral examination evaluated the location bridles upper and lower lip, tongue, structure of the sky, pay attention to the timing of eruption of temporary and permanent teeth, type of occlusion, and tooth position in the dental arch.

RMA - Papillo-marginal-alveolar occlusion is the index of morbidity

of the periodontal tissues.

KPU, KPU+KP, KP - caries-filling-removed tooth -

caries intensity indices.

Analyzing the history of life and diseases of children suffering from type I diabetes, we found that in $28 \%$ of cases they had a hereditary predisposition to this pathology, in $18 \%$ of cases it was provoked by a stressful situation, $43 \%$ of patients noted the first symptoms after a viral infection. $11 \%$ of the observed children and their parents did not associate the onset of the disease with any factor.

Bleeding gums brushing teeth and eating bothered $88 \%$ of children. At the same time, these changes in periodontal tissues often appeared 1.5-2 years before diagnosis. There was hyperemia of the gums, bleeding gums. When examining 
the appearance of the red border and lips, $57 \%$ of children observed changes in the oral cavity in type I diabetes, children did not always complain of hyposalivation. When examining the oral cavity, there was a decrease in the humidity of the oral mucosa. According to the results of CPR, we noted a high intensity of caries in children with this pathology.

The intensity of caries was determined using the KPU+KPU index according to Yu.a. Fedorov and V. V. Volodkina.

It was found that more than half of the patients had a medium-sized face $(52 \%)$, a triangular shape $(41.3 \%)$ and a straight profile $(47.8 \%)$.

Facial asymmetry was observed only in isolated cases (4\%). During the study of the temporomandibular joint, it was found that in most (90\%) cases, opening the mouth was painless, and yet $6.2 \%$ experienced pain, $1.0 \%$ had difficulty opening the mouth. 3.2\% of children had a crunch when moving the lower jaw, and $8.5 \%$ had clicks. Speech formation was found to be satisfactory in only half of the patients $(28.9 \%)$. Speech disorders were $10 \%$ of children - violation of the pronunciation of the letter $\langle\mathrm{p}\rangle, 8.0 \%$ whistling speech, $8.7 \%$-dominated by hissing sounds, $6.2 \%$ - violation of the pronunciation of the letter $\langle 1\rangle, 1.1 \%$ - not clear speech.

\section{Conclusion}

Thus, we can conclude that, despite the fact that, from the very early stages of the diagnosis of diabetes, monitoring and prevention are carried out, the dynamics of the main dental indicators is increasing.

Based on the results of the index assessment of the oral cavity, it can be concluded that children with type I diabetes have a significant significant increase in the values of the CPI index and the hygiene index. The results of a comprehensive examination of the oral cavity in patients with type I diabetes indicate a significantly higher prevalence of major dental diseases.

\section{References}

1. Gunbina I. V. Information technologies in children's diabetology pediatric Bulletin of the southern Urals No. 1, 2017GBUZ CHODKB, Chelyabinsk, Russia

2. Kasatkina E. P. diabetes mellitus in children and adolescents: Manual for doctors/E. P. Kasatkina. Moscow, 1996. p. 224 - 236.

3. Kravets E. B. Diabetology: the scale of the problem, achievements and promising directions Bulletin of Siberian medicine, No. 1, 2005

4. Leus P. N., Garegled A. A., Chudekovai.O., Collection of materials for the IX Russian conference with international participation "Pediatrics and pediatric surgery in the Volga Federal district" (Kazan) Number: 7-2 (63) Year: 2012

5. Naumova V. N., Maslak E. E. the problem of diabetes in real dental practice (results! social research) // Dentistry and socially significant diseases: sat. Tr 10-th vseros. sci.-praktkonf. — SPb: Chelovek, 2013. - Pp. 174-176.

6. Irgashevich, D. A. (2020). Development of national network (tas-ix). ACADEMICIA: An International Multidisciplinary Research Journal, 10 (5), 144-151. 